\title{
Isolation of (-)-Patchouli Alcohol from Patchouli Oil by Fractional Distillation and Crystallization
}

\author{
Zu Qing Su${ }^{1}$, Xiao Li Wu ${ }^{1}$, Mei Jia Bao ${ }^{2}$, Chu Wen $\mathrm{Li}^{1}$, Song Zhi Kong ${ }^{1}$, Zi Ren \\ $\mathrm{Su}^{1,3}$, Xiao Ping Lai ${ }^{1,3}$, Yu Cui $\mathrm{Li}^{{ }^{*}}$ and Jian Nan Chen ${ }^{1,3^{*}}$ \\ ${ }^{1}$ School of Chinese Materia Medica, Guangzhou University of Chinese Medicine, Guangzhou 510006, ${ }^{2}$ Guangzhou \\ Pharmaceutical Vocational School, Guangzhou 511455, ${ }^{3}$ Dongguan Mathematical Engineering Academy of Chinese Medicine, \\ Guangzhou University of Chinese Medicine, Songshan Hu Industrial Park, Dongguan 523808, Guangdong, PR China
}

*For correspondence: Email: chenjiannan@gzucm.edu.cn, liyucui85@163.com; Tel: +86-20-39358517; Fax: +86-20-39358390

Received: 13 August 2012

Revised accepted: 12 January 2014

\begin{abstract}
Purpose: To establish a new and efficient method for the isolation of (-)-patchouli alcohol (PA) from patchouli oil (PO) .

Methods: PO, obtained from commercial source, was separated into four fractions $(A, B, C$ and raffinate) using fractional distillation according to pre-set reflux ratio in vacuum. $P A$ was crystallized from fraction $C$ (containing more than $80 \%$ PA by weight) by cooling and centrifugation. Finally, PA was further purified by suction filtration. Characterization of PA was performed by melting point (MP), infrared spectroscopy (IR), ${ }^{1} \mathrm{H}$ and ${ }^{13} \mathrm{C}$ nuclear magnetic resonance spectroscopy (NMR) and mass spectrometry (MS).

Results: The total yield of PA in this procedure reached $52.9 \%$. The structure of PA was obtained based on data from ${ }^{1} \mathrm{H}-\mathrm{NMR},{ }^{13} \mathrm{C}-\mathrm{NMR}$ and $\mathrm{MS}$ analysis with the aid of literature data for authenticated samples.

Conclusion: Fractional distillation combined with crystallization can be successfully applied to the isolation of PA from $P O$ in solvent-free conditions.
\end{abstract}

Keywords: Isolation, Patchouli alcohol, Patchouli oil, Fractional distillation, Crystallization

Tropical Journal of Pharmaceutical Research is indexed by Science Citation Index (SciSearch), Scopus, International Pharmaceutical Abstract, Chemical Abstracts, Embase, Index Copernicus, EBSCO, African Index Medicus, JournalSeek, Journal Citation Reports/Science Edition, Directory of Open Access Journals (DOAJ), African Journal Online, Bioline International, Open-J-Gate and Pharmacy Abstracts

\section{INTRODUCTION}

(-)-Patchouli alcohol (PA, the chemical structure is shown in Figure 1), is a tricyclic sesquiterpene with a distinct odor of patchouli-like earthy and camphor. Nowadays, PA has been widely used in chemicals, flavors, and perfumery industry. It is used as a fragrance ingredient with a consumption of $0.1-1.0$ metric ton per year [1].

PA is the major constituent of patchouli oil (PO), and there are many methods such as steam distillation [2], supercritical extraction [2] and molecular distillation [3, 4] used in practice for the separation and purification of PA from PO. Nevertheless, all of these methods fail to obtain high-purity PA.

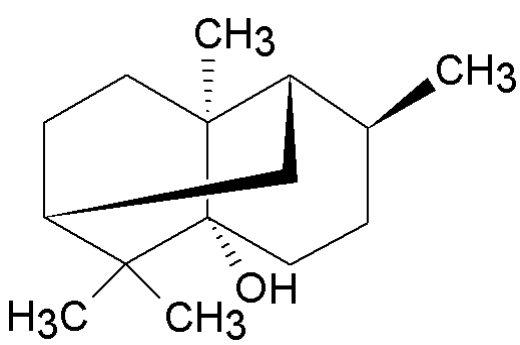

Figure 1: Chemical structure of (-)-Patchouli alcohol 
In addition, methods of silicagel column [5], hostguest inclusion [6] and distillation combining with crystallization [7] had also been developed and successfully applied to the isolation of PA. However, the recovery rates of PA obtained by these methods are lower than $40 \%$, and these methods have many shortcomings, including large energy and/or chemical reagent consumption, solvent residue, low yield, instability and small-scale production.

Therefore, the application of PA has been hampered due to lack of economical and practical methods of manufacture of PA. Consequently, the aim of this study is to develop a large-scale manufacturing method for the isolation of $\mathrm{PA}$ from $\mathrm{PO}$ in solvent-free conditions. Fractional distillation is a highly efficient and solvent-free separation process which depends on the differences of components' boiling points in vacuum. Crystallization is a separation process that depends on the differences in components' freezing point and degree of saturation. Hence, a method combining fractional distillation with crystallization should be feasible and suitable for the isolation of PA from PO.

\section{EXPERIMENTAL}

\section{Material}

Patchouli oil (Lot.120212) was purchased from Guangzhou Baihua Flavours and Fragrances Company Ltd. (Guangzhou, China).

\section{Apparatus}

Separation of PA was carried out with a fractionating tower consisting of a temperature control heating jacket, a round-bottom flask $(1000 \mathrm{~mL})$, a fractionating column $(150 \times 25 \mathrm{~mm}$ inner Diameter) filled with stainless steel Dixon ring packing ( $3 \times 3 \mathrm{~mm}$ inner Diameter), an intelligent thermostat and a reflux condenser. The reflux ratio (reflux flow $(\mathrm{L})$ to distillate flow (D), defined by American Society for Testing and Materials) was controllable and expressed as L/D value. The fractionating tower was operated in vacuum using a PC 101 NT chemistry pumping unit (Vacuubrand $\mathrm{GmbH}$ \& Co. KG, Wertheim, Germany). Distillates were centrifuged with a 216PK table-top refrigerated centrifuge (Sigma Zentrifugen $\mathrm{GmbH}$, Osterode, Germany).

The distillates were determined by as gas chromatography-mass spectrometer (GC-MS) system consisting of an Agilent 6890 gas chromatography instrument, a 5973 mass spectrometer and an Agilent ChemStation software (Agilent, Palo Alto, USA).

\section{Fractional distillation and crystallization procedure}

The PO was placed in the round-bottom flask of fractionating tower, and the pressure of this system was kept at $6 \mathrm{kPa}$. PO was heated at $200.0^{\circ} \mathrm{C}$ until refluxing, and then maintained at $195.0{ }^{\circ} \mathrm{C}$ for 3 h. Subsequently, the concentration of PA in distillates was analyzed by GC-MS every $30 \mathrm{~min}$ while temperature was keep at $215.0{ }^{\circ} \mathrm{C}$. The process flow diagram for $P A$ from $P O$ is shown in Figure 2. According to respective distilled-off temperature, the distillates were designated fraction $A$ (containing no PA), fraction $\mathrm{B}$ (containing $\mathrm{PA}<80 \%$ by weight), fraction $C$ (containing $P A \geq 80 \%$ by weight) and raffinate. Afterwards, fraction $C$ was cool at 15 ${ }^{\circ} \mathrm{C}$ for $10 \mathrm{~min}$, and then centrifuged at $3000 \mathrm{rpm}$ for $10 \mathrm{~min}\left(15^{\circ} \mathrm{C}\right)$. PA crystals were obtained after filtration.

\section{Gas chromatography (GC)-mass spectrometry (MS) analysis}

The distillates diluted with n-hexane (1:6) were analyzed by GC-MS. Chromatographic separation was carried on a $5 \%$ phenyl methyl siloxane HP-5MS capillary column (30 m × 0.25 $\mathrm{mm}$ inner Diameter, $0.25 \mu \mathrm{m}$ film). The oven temperature was set initially at $60^{\circ} \mathrm{C}$, followed by a gradient of $3{ }^{\circ} \mathrm{C} / \mathrm{min}$ up to $140{ }^{\circ} \mathrm{C}$ (held for 1 min), and then programmed to $160{ }^{\circ} \mathrm{C}$ at $1{ }^{\circ} \mathrm{C}$ $/ \mathrm{min}$ (held for $1 \mathrm{~min}$ ), finally, the temperature was up to $230{ }^{\circ} \mathrm{C}$ at $10^{\circ} \mathrm{C} / \mathrm{min}$ (held for $1 \mathrm{~min}$ ). Split injection $(1 \mu \mathrm{L})$ was conducted with a split ratio of $60: 1$ and helium was used as carrier gas with 1.0 $\mathrm{mL} / \mathrm{min}$ flow-rate. The spectrometer was set in electron-impact (EI) mode, the ionization energy was $70 \mathrm{eV}$, the scan range was 50-400 amu and the scan rate was $0.34 \mathrm{~s}$ per scan. The inlet and the ionization source temperatures were 250 and $280{ }^{\circ} \mathrm{C}$, respectively. Identification of the compounds was based on data from Wiley/NBS Registry of Mass Spectral Data (V.5.0) and National Institute of Standards and Technology (NIST) MS Search (2011, V.2.0).

\section{Structure identification}

Melting point (MP) was determined by a B-545 melting point in capillary tubes (Büchi, Switzerland). Optical rotation was measured by a Model 341 Polarimeter (PerkinElmer, USA) and $[\alpha]_{\mathrm{D}}$ value was given in units of $10^{-1}$ deg cm2 g $\mathrm{g}^{-1}$. IR spectra were determined with an Impact 410 spectrometer (Nicolet Company, USA). The spectra were recorded in a transmittance mode 


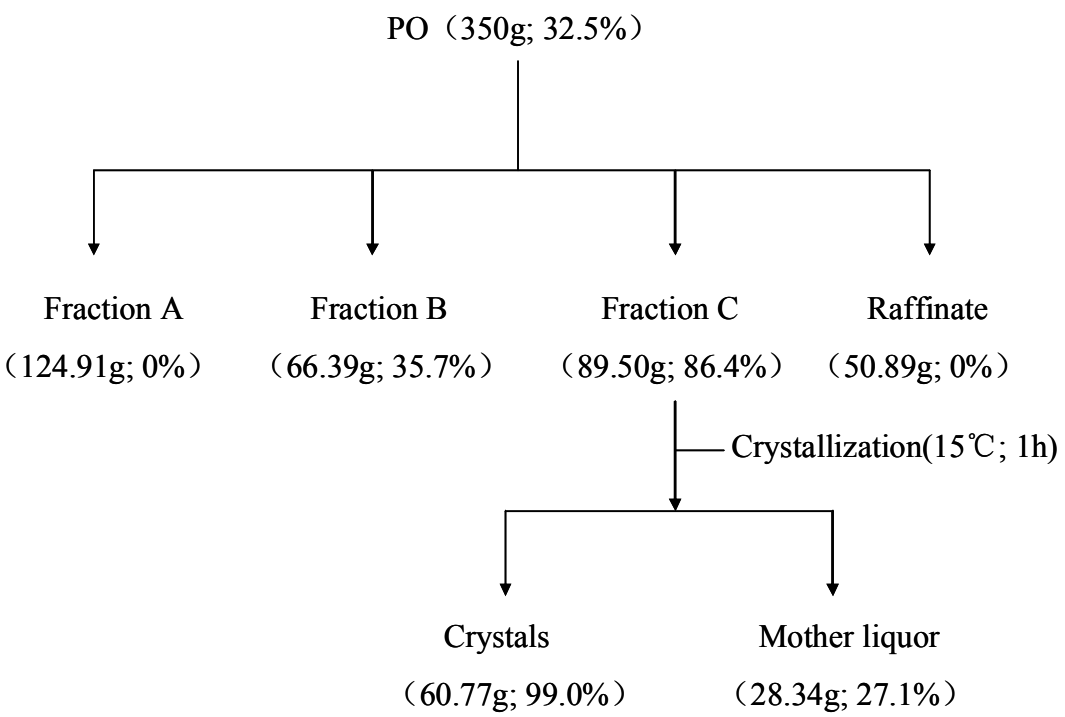

Figure 2: Separation and purification of PA. The yield (weight of patchouli oil, fraction, crystals and mother liquor; concentration of PA)

from 4000 to $400 \mathrm{~cm}^{-1}$ at a resolution of $4 \mathrm{~cm}^{-1}$. ${ }^{1} \mathrm{H} \quad \mathrm{NMR} \quad(300 \mathrm{MHz})$ and ${ }^{13} \mathrm{C}$ NMR $(75$ $\mathrm{MHz}$ )spectra were recorded on JNM EX-400 nuclear magnetic resonance spectrometer ( JEOL Ltd., Japan), $\mathrm{CDCl}_{3}$ was used as a solvent and tetramethylsilane as an internal reference. Chemical shifts $(\delta)$ were expressed in ppm shift. Mass spectra were recorded on MAT TSQ 7000 mass spectrometer using direct inlet (EI) mode (Finngan Company, USA).

\section{RESULTS}

\section{Separation and purification of PA}

The weight and PA content of all fractions described above are shown in Figure 2. By fractional distillation, PO $(350.00 \mathrm{~g}, 32.5 \%)$ was separated into fraction A (124.91g, 0.0\%), fraction B $(66.39 \mathrm{~g}, 35.7 \%)$, fraction C (89.50g, $86.4 \%)$ and raffinate $(50.89 \mathrm{~g}, 0 \%)$. Then fraction C was crystallized to obtain PA crystals (60.77g) with a concentration of $99.0 \%$. PA was white crystalline, and its physicochemical properties and spectra data of IR, EI-MS, ${ }^{1} \mathrm{H}$ NMR and ${ }^{13} \mathrm{C}$ NMR were fairly in accord with experimental results reported in literature [5].

The total yield of PA in this procedure reached $52.9 \%$, and total separation time was about 7.5 $\mathrm{h}$ without any chemical reagents consumption. In contrast, the yield from column chromatography method for manufacture of PA was < $10 \%$ [5]. From the foregoing results, fractional distillation coupled with crystallization is an environmentfriendly method capable of large-scale production to isolate and purify PA from $\mathrm{PO}$.

\section{Chemical composition of all fractions}

To investigate the process of isolation and purification of PA from PO, the chemical compositions of $\mathrm{PO}$ distillates were analyzed by GC - MS. The total ion current (TIC) chromatogram of $\mathrm{PO}$, fraction $(\mathrm{A}, \mathrm{B}, \mathrm{C})$ and $\mathrm{PA}$ was shown in Figure 3. Components with lower boiling points than that of PA was distillated prior to PA and divided into fraction $A$ and fraction $B$ (Figure 3B, 3C), then fraction $C$ consisting mainly of PA was obtained (Figure 3D). At last, as Figure 3E shown, the production of PA was high purity.

\section{Yield of PA from fraction $C$ at different reflux ratios}

As shown in Table 1, yields of PA in fraction $C$ and separation time were measured according to different reflux ratios.

\section{DISCUSSION}

\section{The feasibility of this process}

In the present study, fractional distillation combined with crystallization was successfully applied to the isolation of PA from PO. PO is a commercial flavor, with a mature production technology and market supply. There are some standards for the quality control of $\mathrm{PO}$, and Chinese Pharmacopoeia prescribes that the content of PA in commercial PO should not to be less than $26 \%$ [8]. Therefore, the source of $P O$ is stable and adequate, which is beneficial to the large-scale production of PA from patchouli oil by fractional distillation and crystallization. 

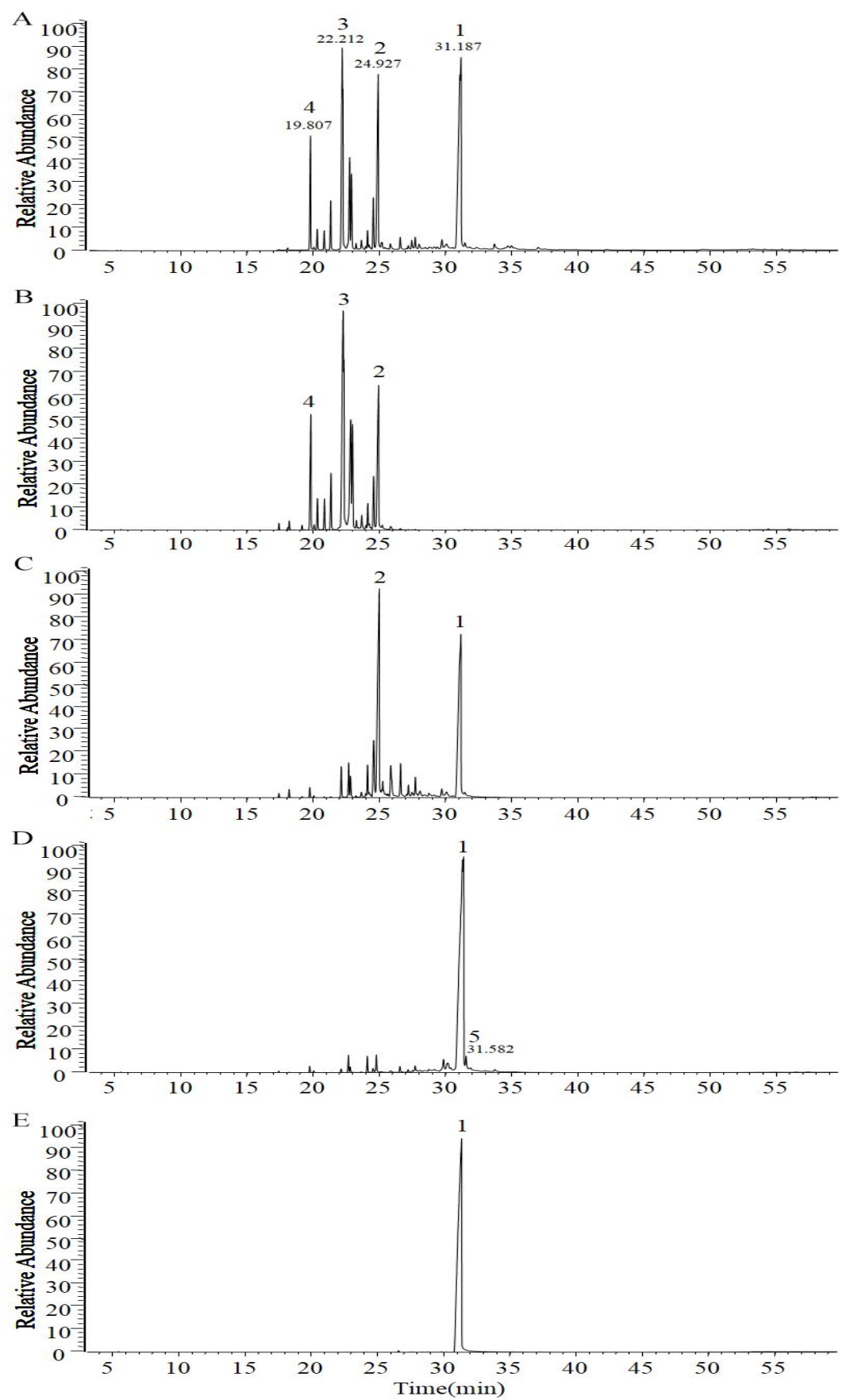

Figure 3: TIC chromatograms of $P O$, fraction $A$, fraction $B$, fraction $C$ and purified $P A$ were shown in $A, B, C, D$ and $E$, respectively. And peak $1,2,3,4,5$ were $P A, \delta$-guaiene, a-longipinene, $\beta$-patchoulene and longipinocarrone, respectively.

Table 1: Yield of PA in fraction $C$ and separation time with different reflux ratios

\begin{tabular}{llll}
\hline No. & Reflux ratio control & $\begin{array}{l}\text { Yield of PA from } \\
\text { fraction C }(\%)\end{array}$ & Separation time (h) \\
\hline 1 & $3: 1^{\mathrm{a}}, 3: 1^{\mathrm{b}}, 3: 1^{\mathrm{c}}$ & 39.7 & 6.0 \\
2 & $3: 1,5: 1,5: 1$ & 40.8 & 8.0 \\
3 & $5: 1,3: 1,7: 1$ & 67.9 & 7.5 \\
4 & $7: 1,4: 1,9: 1$ & 68.1 & 9.5 \\
\hline
\end{tabular}

${ }^{a}$ Reflux ratio as fraction $A$ distilled off; ${ }^{\circ}$ reflux ratio as fraction $B$ distilled off;

${ }^{c}$ the reflux ratio as fraction $C$ distilled off. The yield of PA from fraction $C(\%)$ is the ratio of the amount of PA in fraction $C$ to the amount of of PA in patchouli oil in percentage. 


\section{Physical basis for the separation and purification procedure}

As shown in the total ion current (TIC) chromatogram of $\mathrm{PO}$ (Figure $3 \mathrm{~A}$ ), boiling points of most components of $P O$ such as $\beta$ patchoulene, $\alpha$-longipinene and $\delta$-guaiene are rather lower than that of PA. Fractional distillation is a high efficient and solvent-free separation process, which depends on the differences of components' boiling points in a high vacuum. Consequently, components with lower boiling points than that of PA can been distillated prior to $\mathrm{PA}$, and divided into fraction $\mathrm{A}$ and fraction $\mathrm{B}$ (Figure $3 \mathrm{~B}, 3 \mathrm{C}$ ), then fraction $\mathrm{C}$ consisted mainly of PA was obtained (Figure 3D).

However, as TIC chromatogram of fraction C shows, there are some components such as longipinocarrone which have similar molecular weights and boiling points as PA are difficult to be removed by fractional distillation. However, these components are quite different from PA in their freezing points. Crystallization is a separating process depending on the difference of components' freezing points and degree of saturation. Furthermore, the concentration of PA in fraction $\mathrm{C}$ attained the saturated state at $15{ }^{\circ} \mathrm{C}$ but not for others. Accordingly, PA (Figure 3E) was purified by crystallization at $15{ }^{\circ} \mathrm{C}$ from fraction $\mathrm{C}$.

\section{CONCLUSION}

Natural PA can be isolated from PO under solvent-free conditions using fractional distillation and crystallization technique. The yield of PA, coupled with its purity and the separation time, indicate that this procedure was a more effective and environmentally friendly method, as compared with other methods of synthesis and separation. Furthermore, the aromatic odors of all distillates from PO by this procedure will not be broken and could also be available as fragrance ingredients. In the light of the physical basis for separation of PA, fractional distillation in combination with crystallization can be expected to provide an economical and practical method for the separation and purification of bioactive components from volatile oils.

\section{Authors contribution}

Su $\mathrm{ZQ}$ and $\mathrm{Wu} \mathrm{XL}$ equally contributed to this work. All authors conceived and designed the study, collected and analysed the data as well as prepared and approved the final manuscript for publication.

\section{ACKNOWLEDGEMENT}

The authors thank Mr. Aiwu Zeng of State Key Laboratory of Reaction Engineering in Tianjin University (Tianjin, China) for assistance with fractional distillation. This work was supported by grants from the National Natural Science Foundation of China (Project no. 81173534); The Natural Science Foundation of Guangdong Province of China (Project no. S2012010008893); Guangdong International Cooperation Projects (no. 2012B050300002); Science and Technological Program of Dongguan's Higher Education, Science and Research, and Health Care Institutions (no. 2012105102009).

\section{REFERENCES}

1. Bhatia SP, Letizia CS, Api AM. Fragrance material review on patchouli alcohol, Food Chem Toxicol 2008; 46(11): S255-S256.

2. Liu ZH, Zhou DY. Chemical component comparison of essential oil extracted from Pogostemon cablin by supercritical $\mathrm{CO}_{2}$ extraction and stream distillation. $J$ Anhui Agr Sci 2009; 37(19): 8816-8817.

3. Hu HY, Peng JF, Huang SL, Wu LH, Zhu BZ, Xuan YM, Yang DP. Study on purification technology of patchoul oil with molecular distillation. Chin J Chin Mater Med 2004; 29(4): 320-322, 379.

4. Chen $H$, Zhang JW, Zhu HW, Song ZL. Purification of patchouli alcohol in volatile oil of Pogostemon cablin by molecular distillation. Chin Tradit Herbal Drugs 2009; 40(1): 60-63.

5. Guan L, Quan LH, Xu LZ, Cong PZ. Study on Chemical Constituents of Pogostemon cablin.Chin J Chin Mater Med 1994; 19(6): 355-356.

6. Jian T, Lei Y, Fang G, Wang ZH, Jin L, Guo WS. Selective separation of patchouli alcohol from the essential oil of Cablin potchouli by inclusion crystalline method. Nat Prod Res 2013; 27(1): 32-36.

7.Stefan Berger, Dieter Sicker editors. Classics in Spectroscopy: Isolation and Structure Elucidation of Natural Products. New York: 2009, Wiley-VCH; p. 359-481.

8. The State Pharmacopoeia Commission of the P. R. China, Pharmacopoeia of the People's Republic of China (Vol.1), Beijing: 2010, Chemical Industry Press; pp 42, 373. 